Article - Human and Animal Health

\title{
Verification of the Effects of Red Light-emitting Diode Therapy on Acute Lung Injury in a Sepsis Model in Rats
}

Willer Eduardo Alves ${ }^{1}$

https://orcid.org/0000-0003-4598-0025

\section{Carla Roberta Tim ${ }^{1}$}

https://orcid.org/0000-0002-4745-9375

Cintia Cristina Santi Martignago
https://orcid.org/0000-0003-3980-6354
Richard Liebano ${ }^{2}$

https://orcid. Janaina org/0000-0003-4795-6723

\section{Gomes Rodrigues Santos 1}

https://orcid.org/0000-0002-3557-1909

Lívia Assis ${ }^{1^{*}}$

https://orcid.org/0000-0002-8343-3375

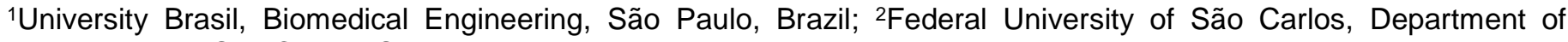
Physiotherapy, São Carlos, São Paulo, Brazil.

Received: 2018.11.19; Accepted: 2019.11.25.

*Correspondence: livinha_fisio@yahoo.com.br; Tel.: +55-11-20700025 (F.L.)

\section{HIGHLIGHTS}

- Sepsis is a significant health care issue.

- Lung is one of the first failure organ induced by sepsis.

- LED is a non-coherent and non-collimed light.

- LED may attenuate lung pathological changes.

Abstract: The aim of this study was to evaluate the in vivo response of red light-emitting diode (LED) on acute lung injury $(A L I)$ in a sepsis model in rats. Twenty rats were randomly allocated into two experimental groups $(n=10)$ : Control Sepsis Group (CS); sepsis and red LED group (SRL). The anterior region of the trachea and ventral regions of the chest (below the ribs), bilaterally were irradiated daily for two consecutive days, starting immediately after the surgery using red $(630 \mathrm{~nm})$ LED. The histological results showed that in red LED treated group presented a modulation of the lung inflammatory process, less intense alveolar septum thickening and decrease of the inflammatory cells. Moreover, LED significantly reduced the lung injury score and increased interleukin type 10 (IL-10) protein expression compared SG. These results suggest that LED was efficient in attenuating ALI in a sepsis model in rats by reducing inflammatory cells into lung tissue and enhancing the anti-inflammatory cytokine production.

Keywords: light-emitting diode (LED); photobiomodulation; sepsis; lung; rehabilitation. 


\section{INTRODUCTION}

Sepsis is a significant health care issue associated with high mortality and healthcare cost [1]. It is characterized by a dysregulated host response to infection with leads to systemic inflammatory response and multiple organ dysfunction syndrome (MODS) $[2,3]$.

Lung is one of the first failure organ induced by sepsis and this failure is the most frequent risk factor for acute respiratory distress syndrome (ARDS), the most severe form of acute lung injury (ALI) [4]. These clinical syndromes are characterized by rapid-onset of respiratory failure following a variety of direct and indirect insults to the parenchyma or vasculature of the lungs $[5,6]$. As the progression can be observed large inflammatory responses, lung oxidative stress associated with diffuse injury to the alveolar epithelium and endothelium allowing the exudation of protein-rich fluid from the blood into the alveolar space, which impairs gas exchange and causes hypoxemic respiratory failure [5]. Clinically, ALI/ARDS is manifested by diffuse alveolar infiltrates, lung edema formation, decreased lung compliance and severe hypoxemia [7].

Furthermore, treatments for reducing these complications are based mainly with the use of broad spectrum antibiotics, corticosteroids, $\beta 2$-Agonists and Surfactant Replacement Therapy [8,9]. However, the mortality of patients from ALI/ARDS remains unacceptably high (20-40\%) [8]. Recently, more innovative therapeutic approaches have been developed aiming to attenuate these lung pathological change caused by sepsis. Among the innumerable range of interventions, the positive effects of photobiomobulation by lowlevel light therapy (LLLT) on lung inflammatory diseases have been highlighted [10-12].

LLLT by lasers is an effective and low cost treatment, able of modulating inflammatory reactions through regulation of pro and anti-inflammatory cytokines and chemokines secretion in several studies of lung disorders [13-15]. However, the optimal parameters for minimizing lung injury in sepsis are not known yet. In particular, there is a lack of studies in the literature investigating the effect of light therapy by Light-emitting diode (LED) on the treatment of lung disorders. LED is a non-coherent and non-collimed light source that is suggested for healing on several tissues by the National Aeronautics and Space Administration (NASA) [16]. Various studies have demonstrated that LED presents good results for those diseases that are characterized by inflammation [17,18]. Although the positive effects of LED, its mechanisms of action are poorly understood.

Despite the stimulatory effects of the LLLT on lung disorders treatment demonstrated by many authors, there is a lack of information about the interaction of LED treatment on the acute lunge injury from sepsis. In this context, it was hypothesized that the LED may attenuate lung pathological changes caused by sepsis in rats was raised, constituting a more suitable and effective treatment to be used. Thus, the aim of this study was to evaluate the effectiveness of red LED on ALI using an experimental model of CLP in rats. For this purpose, morphological aspects of the lung inflammation and the expression of biomarkers of lung injury were evaluated

\section{MATERIAL AND METHODS}

\section{Experimental groups}

Twenty adult male Wistar rats (Rattus norvegicus), weighing $\pm 250 \mathrm{~g}, 6$ weeks old were used in the present study. Animals were placed in plastic cages with sawdust bedding, with three animals per cage and were allowed to move freely in the cages, with free access to commercial food and water. The room had 12 $\mathrm{h}$ dark/light cycle and a controlled temperature $\left(24 \pm 2{ }^{\circ} \mathrm{C}\right)$. This study was approved by the Animal Care Committee guidelines and it was conducted according to the Guiding Principles for the Use of Laboratory Animals. The experimental animals were randomly distributed into two groups ( $n=10$ per group): Control Sepsis group (CS); Sepsis and Red LED group (SRL).

\section{Sepsis experimental model}

Sepsis was produced by cecal ligation and puncture (CLP) as previously described [19]. For the surgical procedure, rats received intra-peritoneal anesthesia with $40 \mathrm{mg} / \mathrm{kg}$ ketamine (Dopalen; Vetbrands; São Paulo; Brazil) and 20 mg/kg xylazine (Anasedan; Vetbrands; São Paulo; Brazil). Afterwards, a midline laparotomy was performed and the cecum was ligated at its base and punctured twice with a 22-Gauge needle. The cecum was then returned to the peritoneal cavity, and this area was prepared and draped using standard sterile surgical technique. Animals were resuscitated with $10 \mathrm{ml}$ of $0.9 \%$ sterile saline solution administered subcutaneously and were housed in single plastic cages and were given appropriate postoperative care. In 
order to minimize post-operative discomfort, the animals received analgesia $(0.2 \mathrm{mg} / \mathrm{kg}$ buprenorphine Temgesic; Reckitt Benckiest Healt Care Ltd. Schering-Plow, Hoddesdon, UK) directly after the operation and subcutaneously (12/12 hours) for two days after surgery.

\section{LED protocol}

LED were performed using a gallium-aluminum-arsenide (InGaAIP) $630 \mathrm{~nm}$ diode led (Ibramed, Brazil) with the following parameters: continuous radiation mode, $200 \mathrm{~mW}$ power output, $20 \mathrm{sec}$ irradiation time, $0.5 \mathrm{~cm}^{2}$ spot area, energy density $8 \mathrm{~J} / \mathrm{cm}^{2}$, irradiance $0,4 \mathrm{~W} / \mathrm{cm}^{2}$ and $4 \mathrm{~J}$ total energy per point. The LED irradiation was performed daily for two consecutive days, starting immediately after the surgery (2 sessions) at 3 points: anterior region of the trachea and on ventral regions of the chest bilaterally (just below the ribs), through the punctual contact technique.

Two days after surgery, all animals were euthanized individually by anesthesic overdose (twofold anesthesic dose) and the lung of each animal was removed for analysis.

\section{Histological procedures and qualitative evaluation}

After surgical resection of the specimens of all experimental group, the right lung was washed immediately with saline and fixed in 10\% buffered formalin (Merck, Darmstadt, Germany) for 12 hours, followed by dehydration in a graded series of ethanol and embedding in paraffin. Thin sections ( $3 \mu \mathrm{m})$ in the longitudinal axis of the lung were obtained using a microtome (Leica Microsystems SP 1600, Nussloch, Germany). Sections of each specimen were stained with hematoxylin and eosin (HE) (Merck) and examined using light microscopy (AxioVision 4.7; Carl Zeiss ${ }^{\circledR}$, Germany). Two experienced observers (LA and WA) performed the qualitative evaluation [20] in a blinded manner.

\section{Lung injury scoring system}

The lung injury in all samples was assessed and compared according to the American Thoracic Society [21]. Sample was assessed on a scale of $0-2$ for each of the following criteria: A) neutrophils in the alveolar space; B) neutrophils in the interstitial space; C) Hyaline membranes; D) Proteinaceous debris filling the airspaces; E) Alveolar septal thickening. The final lung injury score was derived from the following calculation: Score $=[(20 \times A)+(14 \times B)+(7 \times C)+(7 \times D)+2 \times E)] /$ (number of fields $\times 100)$. Increasing score indicates a less lung injury. Digital images of the 400x magnification were captured by optical microscope (Carl Zeiss, Oberkochen, Germany). All variables were measured in 5 fields in each animal and two experienced observers (LA and WA) performed the scoring in a blinded manner.

\section{Morphometric evaluation of inflammatory cells}

The same image analysis technique (Axiovision 4.7; Carl Zeiss, Oberkochen, Germany) was used to count the number of fibers in five preselected areas $\left(500 \mu \mathrm{m}^{2}\right)$ to determine the total inflammatory cells density (polymorphonuclear and mononuclear). Two experienced observers (LA and WA) performed the scoring in a blinded manner.

\section{Immunohistochemistry analysis}

For iNOS and IL-10 expression analysis the paraffin was removed with xylene from serial sections of 5 $\mu \mathrm{m}$. After this procedure, the sections were rehydrated in graded ethanol and pretreated in a microwave with $0.01 \mathrm{M}$ citric acid buffer $(\mathrm{pH} 6)$ for 3 cycles of $5 \mathrm{~min}$ each at $850 \mathrm{~W}$ for antigen retrieval. Subsequently, the material was pre-incubated with $0.3 \%$ hydrogen peroxide in phosphate-buffered saline (PBS) solution for 5 min to inactivate the endogenous peroxidase and then blocked with $5 \%$ normal goat serum in PBS solution for $10 \mathrm{~min}$. The specimens were incubated with anti-iNOS polyclonal primary antibody (Santa Cruz Biotechnology, USA; SC-651) at a concentration of 1:50 and anti-IL-10 polyclonal primary antibody (Santa Cruz Biotechnology, USA; sc-1783) at a concentration of 1:200. Incubation was performed overnight at $4^{\circ} \mathrm{C}$ in the refrigerator and followed by two washes in PBS for 10 min. Afterwards, the sections were incubated with biotin conjugated secondary antibody anti-rabbit IgG (Vector Laboratories, Burlingame, CA, USA) at a concentration of 1:200 in PBS for $1 \mathrm{~h}$. The sections were washed two times with PBS followed by the 
application of avidin biotin complex conjugated to peroxidase for $45 \mathrm{~min}$. The visualization of the bound complexes was realized by the application of a $0.05 \%$ solution of $3-3$ '-diaminobenzidine solution and counterstained with Harris Hematoxylin. Finally, for control analyses of the antibodies, the serial sections were treated with rabbit IgG at a concentration of 1:200 instead of the primary antibody. Furthermore, internal positive controls were performed with each staining bath. Digital images of the 200x magnification were captured by optical microscope. The results were evaluated both qualitatively (presence of the immunomarkers) and semi quantitatively according to a previously described scoring scale from 1 to 4 ( 1 = absent, 2 = weak, 3 = moderate, and 4 = intense for immunohistochemical analysis). Two experienced observers (WA and LA) performed the scoring in a blinded manner.

\section{Statistical Analysis}

Data are expressed as the mean \pm standard error of the mean (SEM). Shapiro-Wilk's and Levene's test were applied to evaluate the normality and homogeneity of the results, respectively. For the variables that exhibited normal distribution, comparisons between experimental groups were performed by Student's t-test. For the variables that exhibited non-normal distribution, Mann-Whitney test was used. A $P$ value $<0.05$ was considered significant. All analyzes were performed using a GraphPad Prism 6.0 (GraphPad Software, San Diego CA, USA).

\section{RESULTS}

\section{General observation of the experimental animals}

From the 20 animals available for this study, two animals were lost after CLP surgery. The remaining 18 animals recovered uneventfully from the CLP induction and LED protocol. The survival estimate was 0.9 and the mean survival was 1.8 days in both groups. No signs of macroscopic adverse tissue responses were observed during the experimental period.

\section{Histological descriptive analysis}

Representative images of the lung tissue sections are shown in Figure 1. Histopathological analysis revealed that 3 days post-surgery, SC group demonstrated severe morphological modifications, characterized by edema in the interstitial area, thickening of the alveolar septum and massive cellular inflammatory infiltration. For the SRL group, also morphological modifications was observed however less intense alveolar septum thickening and inflammatory cells recruitment in the lung compared to SC group.

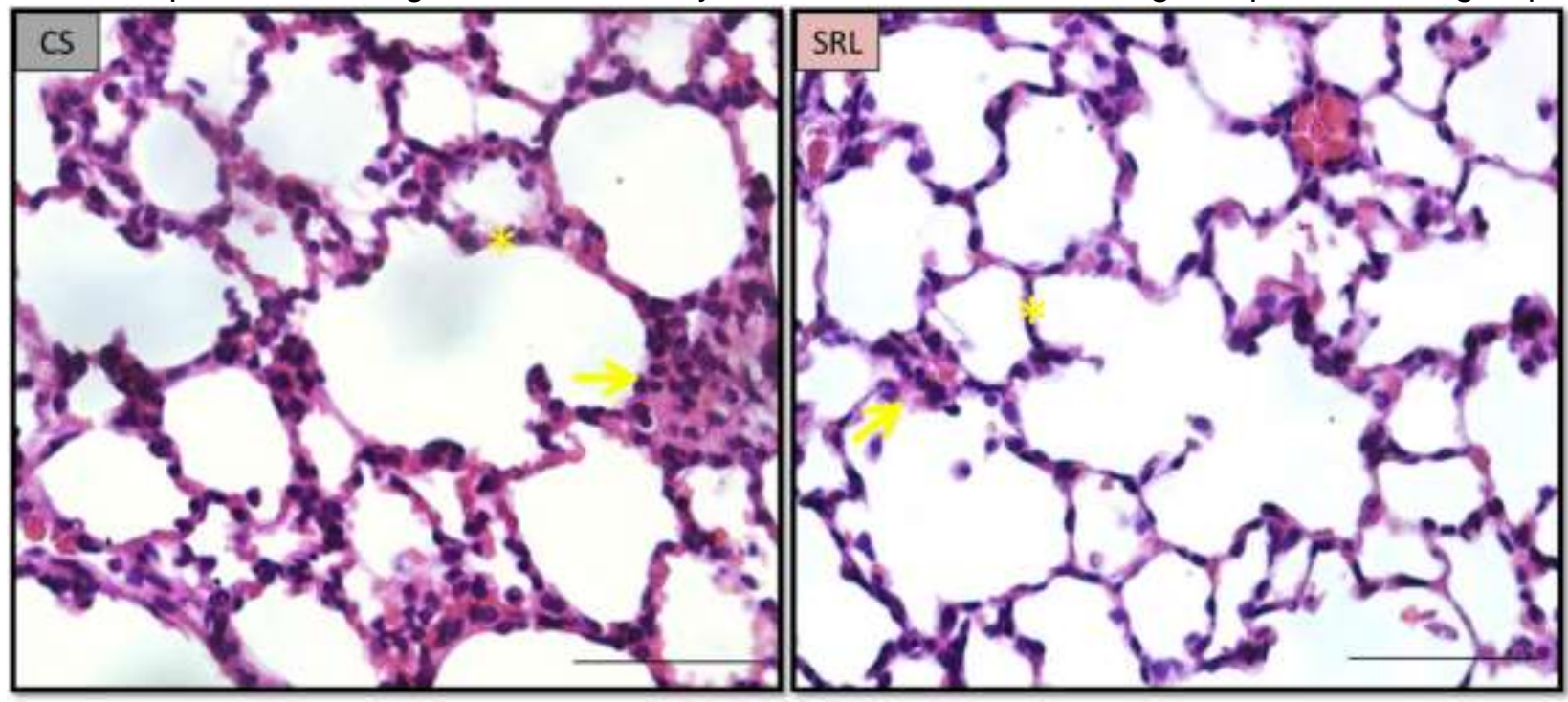

Figure 1. Representative photomicrographs of morphological analysis of acute lung injury cross-sections. Alveolar septum (asterisks); presence of inflammatory process (arrow). Control Sepsis group (CS); Sepsis red LED group (SRL). (Stain: HE; Scale bar: $20 \mu \mathrm{m}$ ). 


\section{Lung injury score}

The score of lung injury was significantly lower in SLR $(p=0.0077)$ compared to CS (Figure 2$)$.

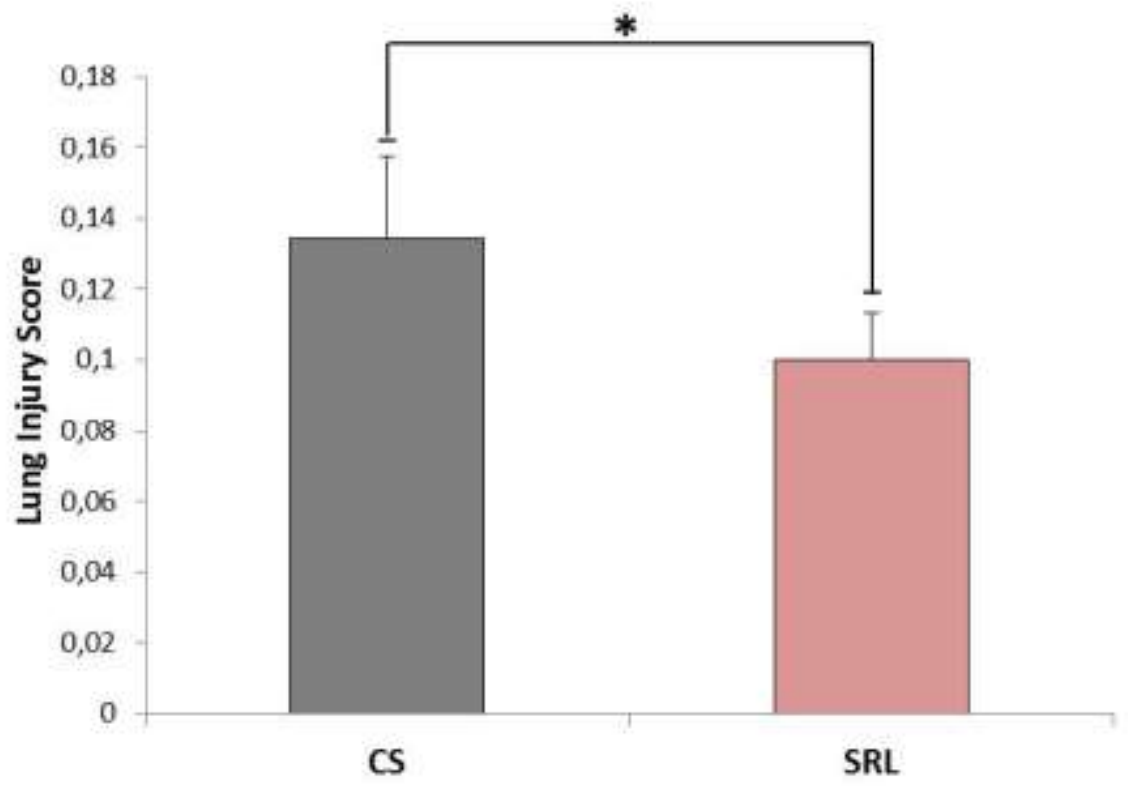

Figure 2. Lung injury scoring system analysis. Control Sepsis group (CS); Sepsis red LED group (SRL). (indicated as ${ }^{*} p=0.0077$ versus CS; $n=18$; Mann-Whitney test).

\section{Density of Inflammatory cells}

Figure 3 shows the morphometric evaluation of the density of inflammatory cells. The percentage of inflammatory cells was significantly higher in SC compared to SLR ( $p=0.0022)$.

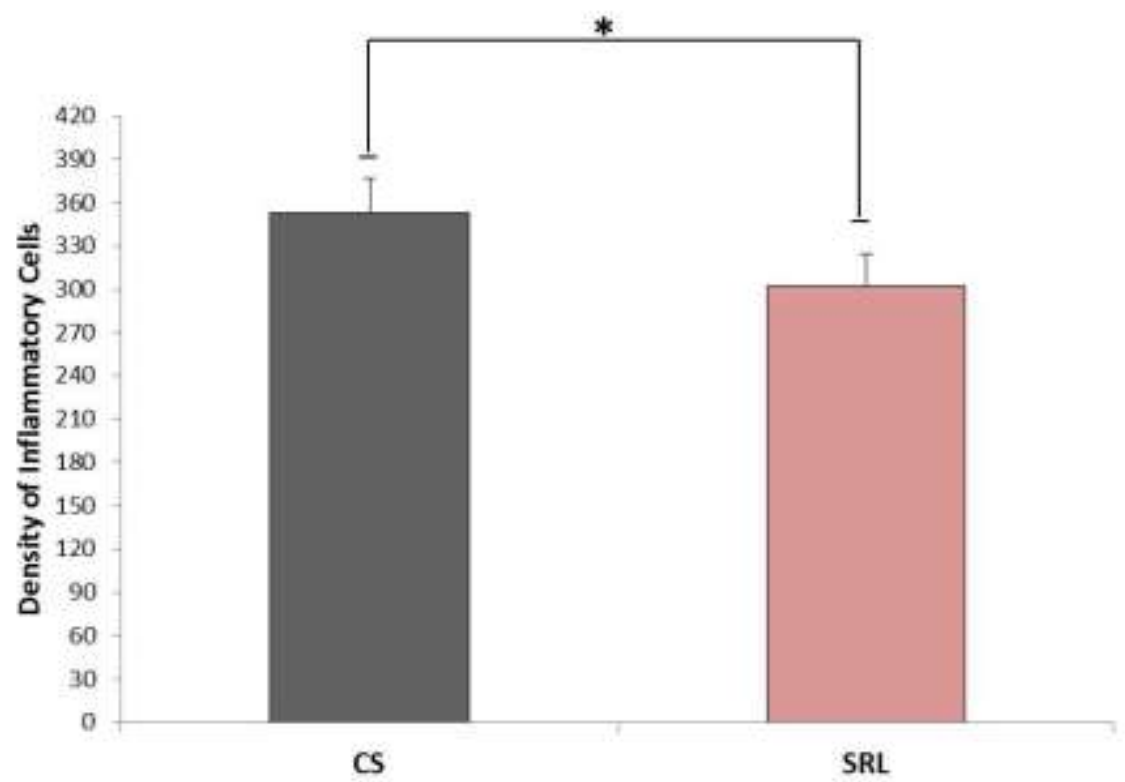

Figure 3. Morphometric analysis of density of inflammatory cells. Control Sepsis group(CS); Sepsis red LED group (SRL). (indicated as ${ }^{*} p=0.0013$ versus IR; $\# p=0.0022$ versus $C S ; n=18$; Mann-Whitney test). 


\section{Immunohistochemistry analysis}

\section{iNOS expression}

The immunohistochemistry results revealed positive iNOS staining in the inflammatory cells in all groups (Figure 4A). However, the semi-quantitative analysis demonstrated no difference in iNOS immunoexpression among groups (Figure 4B).

A)

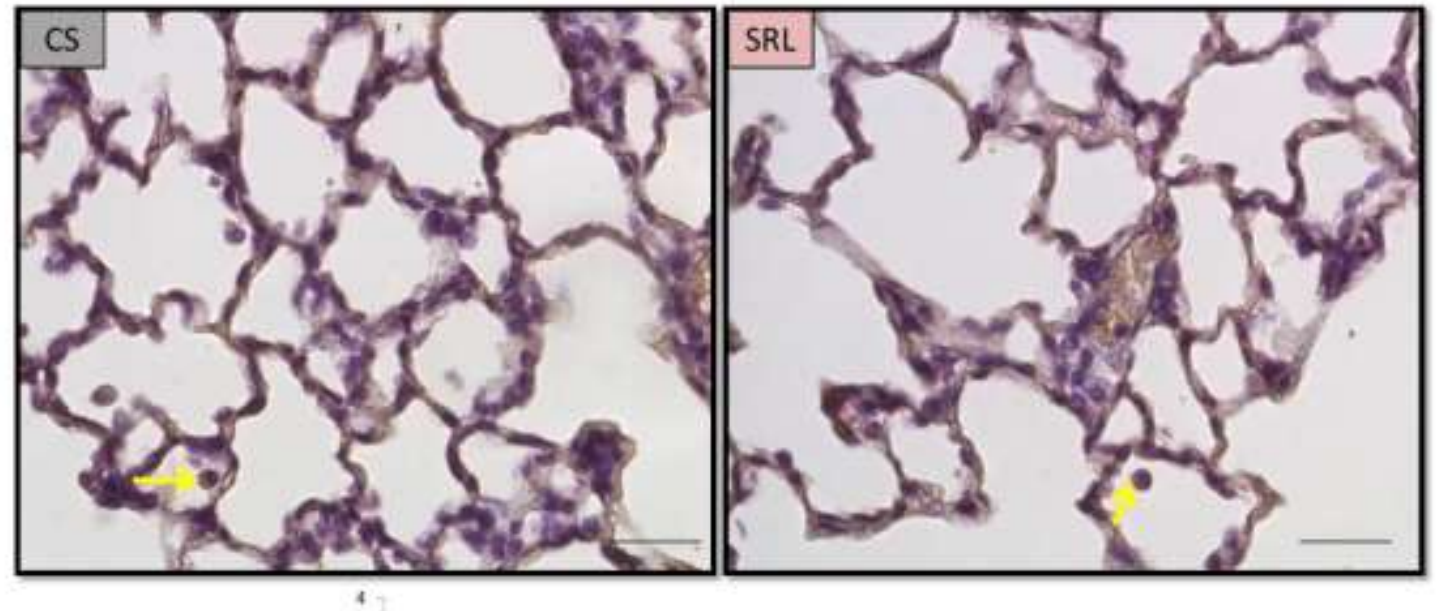

B)

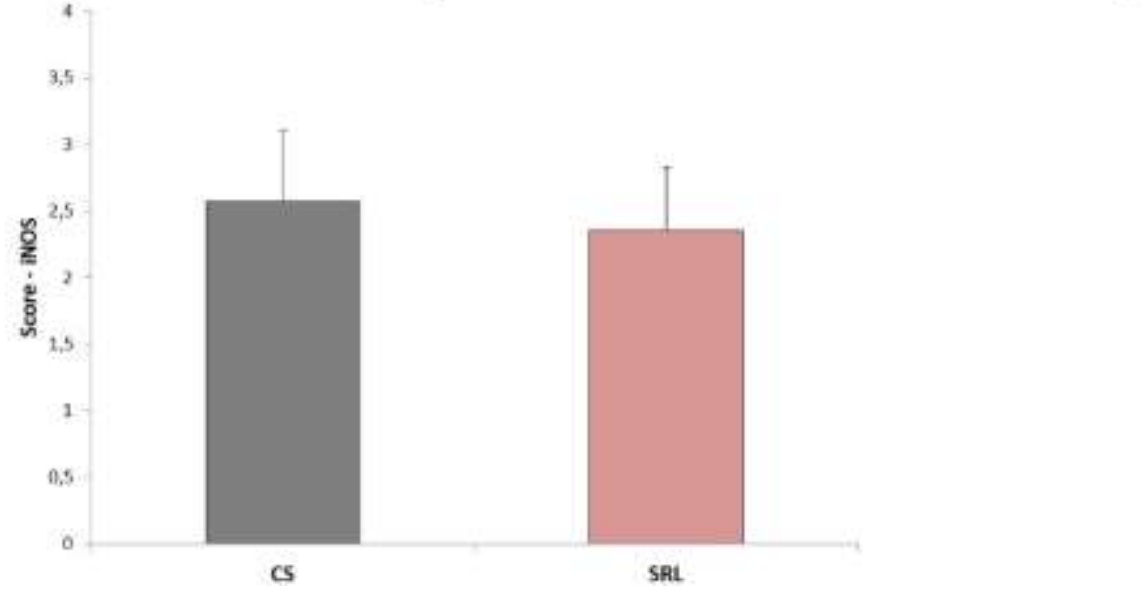

Figure 4. A) Representative sections of iNOS immunohistochemistry. Immunolabeled inflammatory cells (arrow). B) Results of semi-quantitative analysis of the iNOS expression. Control Sepsis group (CS); Sepsis red LED group (SRL). ( $\mathrm{n}=18$; Mann-Whitney test).Scale bar: $20 \mu \mathrm{m}$

\section{IL-10 expression}

Immunohistochemistry evaluation demonstrated that IL-10 expression was observed mainly in the inflammatory cells for all groups (Figure 5A). Furthermore, the semi-quantitative analysis exhibited a higher $\mathrm{IL}-10$ expression in SRL compared to SC $(p=0.0036$; Figure $5 B)$. 
A)

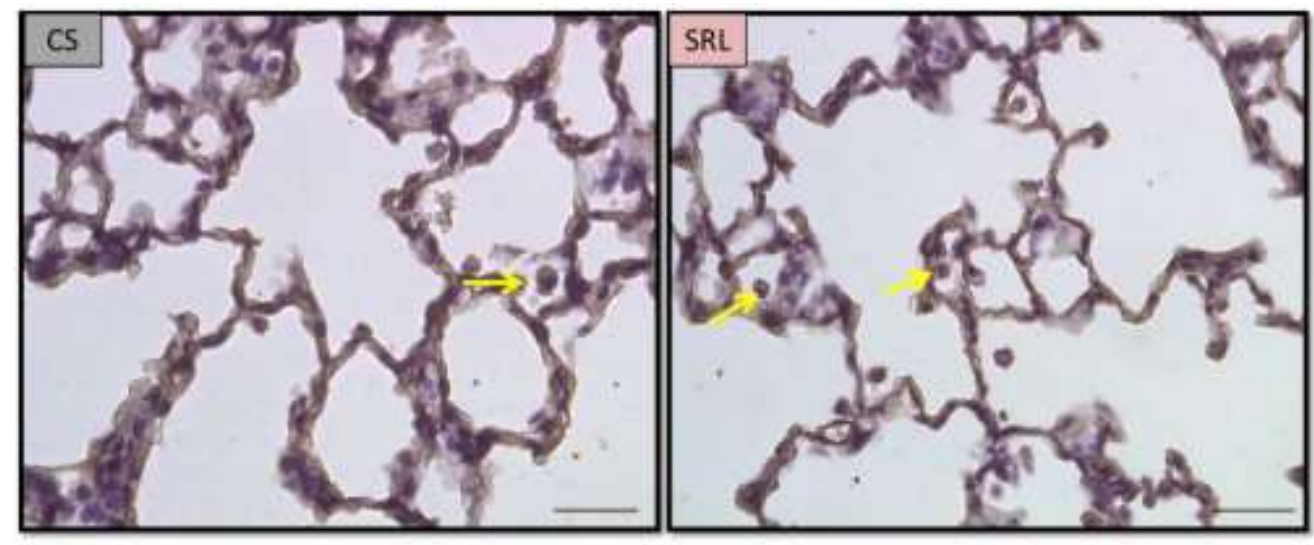

B)

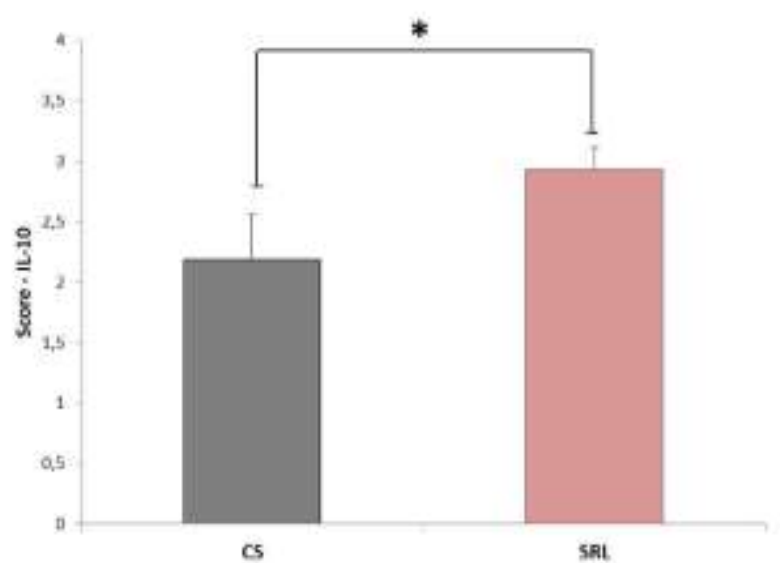

Figure 5. A) Representative sections of IL-10 immunohistochemistry. Immunolabeled inflammatory cells (arrow). Results of semi-quantitative analysis of the IL-10 expression. Control Sepsis group(CS); Sepsis red LED group (SRL). (indicated as * $p=0.0036$ versus SC; $n=18$; Mann-Whitney test). Scale bar: $20 \mu \mathrm{m}$.

\section{DISCUSSION}

This study aimed to evaluate in vivo response of red LED on ALI in a sepsis model in rats. The histological findings demonstrated that LED therapy produced stimulatory effects on modulation of the inflammatory process, less intense alveolar septum thickening and decrease of the density of inflammatory cells. Furthermore, the LED produced a significant reduction of the lung injury score and higher IL-10 expression compared with control sepsis group.

It is of common knowledge sepsis is a potentially life-threatening complication of an infection that leads to impairments in different system function, putting a series of organs at risk, including liver, kidneys and lungs [22,23].

In this context, the LED has been considered a promising alternative to treat many inflammatory diseases due its positive effects on the inflammatory response and repair process [24,25]. The histological findings of the present study are in agreement those statements and showed that LED treatment used induced a modulation of the inflammatory process in the parenchyma and vasculature pulmonary. Also, the morphometric evaluation demonstrated that the red LED irradiated animals presented a lower lung injury score when compared untreated animals. Siqueira and coauthors [24] also observed that red LED (660 nm) decreased the lung inflammatory infiltration in an experimental model of asthma. In addition, in earlier studies of our group using phototherapy in lung inflammation model induced by CLP we showed similar effects of phototherapy in the reduction of inflammatory cells of the lung [26]. It may be suggested that the energy offered to the lung tissue was able of inducing an appropriate tissue-response, modulating the inflammatory process. Therefore, the down-regulation of inflammatory cells recruitment evidenced in the present study may decrease of pulmonary parenchymal damage, consequently, attenuate the lung injury after sepsis.

Additionally, studies have shown that sepsis is associated with enhanced nitric oxide synthase (iNOS) expression by several pro-inflammatory factors [27]. It is known that iNOS induces nitric oxide (NO) overproduction, contributing to sepsis severity. Previously, Costa Carvalho and coauthors [28] demonstrated that $660 \mathrm{~nm}$ wavelenght reduced the production iNOS mRNA expression as well as the NOS activity in allergic 
lung inflammation. Interestingly, in the present study, no effect on the expression of this immunomarker was found with the LED treatment. Possibly, the LED parameters used in the present study were not able to offer sufficient energy to modulate iNOS expression in the experimental period studied.

Furthermore, in the present study, red LED presented an increased IL-10 expression. IL-10 has been widely reported as an important regulatory cytokine that is involved in large number of cellular actives in sepsis [29]. In particular, there is evidence indicating that IL-10 is a potent anti-inflammatory mediator that plays a crucial role in limiting host immunopathology during sepsis by controlling effector T cell activation [30]. Though, previous findings showing that several oxidative exposure is responsible by a down-regulation of this cytokines, resulting amplification of lung inflammatory response [29]. In the current study, red LED was able of increasing the expression of this cytokine compared to control group. Recently, LED has shown promise in modulating the growth factors and cytokines expression related to lung injuries in several treatment parameters of LED [25]. Siqueira and coauthors [25]. demonstrated that the red LED was able to ameliorates allergic lung inflammation, with the up-immunoexpression of IL-10 and others gene expression in in experimental model of asthma. Using the same sepsis experimental model, de Assis and coauthors [32] found a down-regulation of IL-10 immunoexpression after of LLLT irradiation at an early stage of the sepsis process ( 2 days after sepsis induction). Thus, the modulated expression of IL-10 after red LED irradiation may promote anti-inflammatory effects by the reestablishment of homeostasis, increasing the defense mechanisms, contributing to the acceleration of lung injury recovery.

\section{CONCLUSION}

The findings of the present study would suggest that LED was efficient in attenuating sepsis-induced ALI in rats, with involving reduction of inflammatory cells into lung tissue and enhance the anti-inflammatory cytokine production leading to decrease in lung injury. This study may provide important information about the effects of LED, and in addition, it may open the possibility of a new approach for the treatment of ALI in a sepsis.

Funding: This research was funded by CAPES - Coordenação de Aperfeiçoamento de Pessoal de Nível Superior.

Acknowledgments: We acknowledge lbramed for tecnical support.

Conflicts of Interest: The authors declare no conflict of interest.

\section{REFERENCES}

1. Yuki K, Murakami N. Sepsis pathophysiology and anesthetic consideration. Cardiovasc Hematol Disord Drug Targets. 2015;15(1):57-69.

2. Singer M, Deutschman CS, Seymour CW, Shankar-Hari M, Annane D, Bauer M, et al. The Third International Consensus Definitions for Sepsis and Septic Shock (Sepsis-3). JAMA. 2016; 23;315(8):801-10.

3. Rossaint J, Zarbock A. Pathogenesis of Multiple Organ Failure in Sepsis. Crit Rev Immunol. 2015;35(4):277-91.

4. Rubenfeld GD, Caldwell E, Peabody E, Weaver J, Martin DP, Neff M, et al. Incidence and outcomes of acute lung injury. N Engl J Med. 2005;353(16):1685-93.

5. Ware LB, Matthay MA. The acute respiratory distress syndrome. N Engl J Med.

6. 2000 May 4;342(18):1334-49. Review.

7. Bernard G. Acute Lung Failure - Our Evolving Understanding of ARDS. N Engl J.Med. 2017;377(6):507-9.

8. Mokra D, Kosutova P. Biomarkers in acute lung injury. Respir Physiol Neurobiol. 2015;209:52-8.

9. Varisco BM. The pharmacology of acute lung injury in sepsis. Adv Pharmacol Sci. 2011;2011:254619.

10. Sadowitz B, Roy S, Gatto LA, Habashi N, Nieman G. Lung injury induced by sepsis: lessons learned from large animal models and future directions for treatment. Expert Rev Anti Infect Ther. 2011;9(12):1169-78.

11. Miranda da Silva C, Peres Leal M, Brochetti RA, Braga T, Vitoretti LB, Saraiva Câmara NO, et al. Low Level Laser Therapy Reduces the Development of Lung Inflammation Induced by Formaldehyde Exposure. PLoS One. 2015;10(11):e0142816.

12. Oliveira MC Jr, Greiffo FR, Rigonato-Oliveira NC, Custódio RW, Silva VR, Damaceno-Rodrigues NR, et al. Low level laser therapy reduces acute lung inflammation in a model of pulmonary and extrapulmonary LPS-induced ARDS. J Photochem Photobiol B. 2014;134:57-63.

13. Mafra de Lima F, Villaverde AB, Salgado MA, Castro-Faria-Neto HC, Munin E, Albertini R, et al. Low intensity laser therapy (LILT) in vivo acts on the neutrophils recruitment and chemokines/cytokines levels in a model of acute 
pulmonary inflammation induced by aerosol of lipopolysaccharide from Escherichia coli in rat. $\mathrm{J}$ Photochem Photobiol B. 2010;101(3):271-8.

14. Costa Carvalho JL, de Brito AA, de Oliveira AP, de Castro Faria Neto HC, Pereira TM, de Carvalho RA, et al. The chemokines secretion and the oxidative stress are targets of low-level laser therapy in allergic lung inflammation. J Biophotonics. 2016;9(11-12):1208-21.

15. Sergio LPDS, Thomé AMC, Trajano LADSN, Mencalha AL, da Fonseca AS, de Paoli F. Photobiomodulation prevents DNA fragmentation of alveolar epithelial cells and alters the mRNA levels of caspase 3 and Bcl-2 genes in acute lung injury. Photochem Photobiol Sci. 2018;17(7):975-83.

16. Cury V, de Lima TM, Prado CM, Pinheiro N, Ariga SK, Barbeiro DF, et al. Low level laser therapy reduces acute lung inflammation withoutimpairing lung function. J Biophotonics. 2016;9(11-12):1199-207.

17. Whelan HT, Smits RL Jr, Buchman EV, Whelan NT, Turner SG, Margolis DA, et al. Effect of NASA light-emitting diode irradiation on wound healing. J Clin Laser Med Surg. 2001;19(6):305-14.Review.

18. Dall Agnol MA, Nicolau RA, de Lima CJ, Munin E. Comparative analysis of coherent light action (laser) versus noncoherent light (light-emitting diode) for tissue repair in diabetic rats. Lasers Med Sci. 2009;24(6):909-16.

19. Casalechi HL, Nicolau RA, Casalechi VL, Silveira L Jr, De Paula AM, Pacheco MT. The effects of low-level light emitting diode on the repair process of Achilles tendon therapy in rats. Lasers Med Sci. 2009;24(4):659-65.

20. Lang $\mathrm{CH}$, Frost RA, Vary TC. Regulation of muscle protein synthesis during sepsis and inflammation. Am J Physiol Endocrinol Metab. 2007;293(2):E453-9.

21. Zhai $Y$, Zhou X, Dai Q, Fan $Y$, Huang $X$. Hydrogen-rich saline ameliorates lung injury associated with cecal ligation and puncture-induced sepsis in rats. Exp Mol Pathol. 2015;98(2):268-76.

22. Matute-Bello G, Downey G, Moore BB, Groshong SD, Matthay MA, Slutsky AS, et al. Acute Lung Injury in Animals Study Group. An official American Thoracic Society workshop report: features and measurements of experimental acute lung injury in animals. Am J Respir Cell Mol Biol. 2011;44(5):725-38

23. Pierrakos C, Vincent JL. Sepsis biomarkers: a review. Crit Care. 2010;14(1):R15.

24. Yin K, Wilmanski J, Wang C, Qiu G, Tahamont M. Lung compartmentalization of inflammatory cells in sepsis. Inflammation. 2000;24(6): 547-57.

25. Siqueira VPC, Evangelista MIS, Dos Santos A, Marcos RL, Ligeiro-de-Oliveira AP, Pavani C, et al. Light-Emitting Diode treatment ameliorates allergic lung inflammation in experimental model of asthma induced by ovalbumin. $J$ Biophotonics. 2017;10(12):1683-93.

26. Costa SG, Barioni ÉD, Ignácio A, Albuquerque J, Câmara NOS, Pavani C, et al. Beneficial effects of Red LightEmitting Diode treatment in experimental model of acute lung injury induced by sepsis. Sci Rep. 2017;7(1):12670.

27. Bublitz C, Assis L, Rennó AC, Cury V, Harada G, Hirai AH, et al. Laser therapy, used in a specific dose, modulates pulmonary inflammatory processes in an experimental model of sepsis in rats. Fisioterapia e Pesquisa; 2015;22(4), 420-25.

28. Prauchner CA. Oxidative stress in sepsis: Pathophysiological implications justifying antioxidant co-therapy. Burns. 2017;43(3):471-85.

29. Costa Carvalho JL, de Brito AA, de Oliveira AP, de Castro Faria Neto HC, Pereira TM, de Carvalho RA, et al. The chemokines secretion and the oxidative stress are targets of low-level laser therapy in allergic lung inflammation. J Biophotonics. 2016;9(11-12):1208-21.

30. Hotchkiss RS, Moldawer LL, Opal SM, Reinhart K, Turnbull IR, Vincent JL.Sepsis and septic shock. Nat Rev Dis Primers. 2016;2:16045.

31. Leech JM, Lacey KA, Mulcahy ME, Medina E, McLoughlin RM. IL-10 Plays Opposing Roles during Staphylococcus aureus Systemic and Localized Infections. J Immunol. 2017;198(6):2352-65.

32. Assis L, Renno AC, Bublitz C, Hirai AH, Harada G, Kido H et al.Laser therapy modulates systemic inflammatory processes and muscle atrophy in an experimental model of sepsis in rats. Photon Lasers Med. 2015; 4(3): $241-$ 51.

(C) 2020 by the authors. Submitted for possible open access publication under the terms and conditions of the Creative Commons Attribution (CC BY NC) license (https://creativecommons.org/licenses/by-nc/4.0/). 